\title{
Two Fixed Point Theorems in Topological vector space Valued Cone Metric Spaces with Complete Topological Algebra Cones
}

\author{
Tadesse Bekeshie ${ }^{1}$, G.A Naidu ${ }^{2}$ and K.P.R Sastry ${ }^{3}$ \\ ${ }^{1,2}$ (Department of Mathematics Andhra University, Visakhapatnam-530 003, India, \\ ${ }^{3}$ (8-28-8/1, Tamil Street, Chinna Waltair, Visakhapatnam-530 017, India,
}

\begin{abstract}
In this paper we prove two fixed point theorems in topological vector space valued cone metric spaces (briefly TVS-CMS). To that end we introduce the concept of complete topological algebra cone (briefly CTA cone). Our theorems are generalizations of corresponding theorems in [8] and [13]. The paper also gives answers to the open problems posed in [15]. Finally we give an application of our first theorem.
\end{abstract}

AMS Mathematics Subject Classification (2010): 47H10, 54H25, $46 \mathrm{~A} 40$

Key terms: topological vector space, ordered topological vector space, algebra over a field, topological algebra over a field, topological vector space valued cone metric space, scalarization function, CTA cone

\section{Introduction}

The famous Banach Contraction Principle states that every contraction in a complete metric space has a unique fixed point. It has two core hypotheses: completeness and contractivity. Both notions depend on the definition of the underlying metric. Much recent work has focused on the extension of the notion of metric spaces and the related notion of contractivity. One extension of metric spaces is the so called cone metric space. In cone metric spaces, the metric is no longer a positive number but a vector, in general an element of a Banach space equipped with a cone. An overview of a historical background of metric spaces and cone metric spaces is given in the next few paragraphs.

In 1906, the French mathematician Maurice Frechet [4, 6] introduced the concept of metric spaces, although the name "metric" is due to Hausdorff[4, 7].

In 1934, the Serbian mathematician Duro Kurepa[10], a PhD student of Frechet, introduced metric spaces in which an ordered vector space is used as the codomain of a metric instead of the set of real numbers. In the literature the metric spaces with vector valued metrics are known under various names such as pseudometric spaces, k-metric spaces, generalized metric spaces, cone-valued metric spaces, cone metric spaces, abstract metric spaces and vector valued metric spaces. Fixed point theory in K-metric spaces was developed by A.I.Perov in 1964[11, 12]. For more details on fixed point theory in K-metric and K-normed spaces, we refer the reader to [18].

In 2007, Huang and Zhang [8] reintroduced such metric spaces under the name cone metric spaces without mentioning the previous works. But they went further, defining convergent and Cauchy sequences in terms of interior points of the underlying cone. They also proved some fixed point theorems in such spaces in the same work. Subsequently, several authors have studied fixed point theory of cone metric spaces.

In 2009 I.Beg et al [3] and in $2010 \mathrm{Du}$ [5] generalized cone metric spaces to topological vector space valued cone metric spaces (TVS-CMS). In this approach ordered topological vector spaces are used as the codomain of the metric, instead of Banach spaces. While I.Beg et al used Hausdorff TVS; Du used locally convex Hausdorff TVS. However, a result in [16] shows that if the underlying cone of an ordered TVS is solid and normal it must be an ordered normed space. So, proper generalizations from Banach space valued cone metric spaces to TVS-CMS can be obtained only in the case of nonnormal cones.

Several authors had showed that some fixed point theorems in usual metric spaces and their TVS-CMS counterparts are equivalent. See [2], [3] and [5]. One of the tools used to prove such equivalences is the so called nonlinear scalarization function. This method was used earlier in optimization theory to convert optimization problems in vectorial forms to their scalar forms. This function was introduced into fixed point theory by $\mathrm{Du}[5]$.

Our objectives are as follows. We introduce the notion of complete topological algebra (CTA) cone. Then we prove Banach's fixed point theorem and Kannan's fixed point theorem in TVS-CMS with CTA cones. The contraction constants used are taken to be elements of the CTA cone, instead of being scalars. The results obtained generalize those in [8] and [13] hence the classical Banach's and Kannan's fixed point theorems. More over our theorems answer the open problems raised in [15].

In line with the methods used in [2] and [5], we used the nonlinear scalarization method of Du to prove our theorems. This means that we converted our theorem statements (which are given in TVS-CMS (X, d)) to equivalent statements in the metric space $\left(X, d_{e}\right)$ where the metric $d_{e}$ is defined by $d_{e:}=\xi_{e} o$ d while 
Two Fixed Point Theorems in Topological vector space Valued Cone Metric Spaces with Complete $\xi_{e}: \mathrm{E} \rightarrow \mathbb{R}$ given by $\xi_{e}(\mathrm{y}):=\inf \{\mathrm{t} \in \mathbb{R} \mid y \leq t e\}$, e being any element in int $\mathrm{P}$. After converting the statements from "vectorial" form (TVS-CMS) to equivalent statements in "scalar" form (metric spaces $\left(\mathrm{X}, \mathrm{d}_{\mathrm{e}}\right)$ ), then we used known results available in the literature of metric fixed point theory to complete the proofs. We also used a new property of the function $\xi_{e}$ (See Lemma 3.2) in our arguments.

The paper is organized as follows. In section 2 , we consider the notations, notions and known results which we use later in sections 3, 4 and 5. In section 3, we present our main results. The fourth section presents an application and the fifth a conclusion.

\section{Preliminaries}

Definition 2.1[1, 17]: A vector space $E$ over a field $K(\mathbb{R}$ or $\mathbb{C}$ ) is said to be a topological vector space (TVS) over $\mathrm{K}$ if it is furnished with a topology $\tau$ such that the vector space operations are continuous, i.e.,

i) The addition operation $(x, y) \rightarrow x+y$ as a function from $E \times E$ to $E$ is continuous.

ii) The scalar multiplication operation $(a, x) \rightarrow a . x$ as a function from $K \times E$ to $E$ is continuous.

In this case one says that $\tau$ is a vector topology or a linear topology on the vector space $\mathrm{E}$, or that $\tau$ is compatible with the linear structure of $\mathrm{E}$.

Here, $\mathbb{R}$ and $\mathbb{C}$ are equipped with their usual topologies where as $E \times E$ and $K \times E$ are endowed with their respective product topologies.

Caution: Some authors (e.g., Rudin [14]) require the topology on a TVS $E$ to be Hausdorff, an approach which we do not follow in this manuscript.

Definition 2.2 [3, 5]: Let $(E, \tau)$ be a topological vector space. A subset $P$ of $E$ is called a cone if:

i) $\quad \mathrm{P}$ is closed, nonempty and nontrivial (i.e., $P \neq\{0\}$ );

ii) $a x+b y \in P$ for all $x, y \in P$ and nonnegative real numbers $a$ and $b$ and

iii) $P \cap(-P)=\{0\}$.

If, in addition, the interior of $\mathrm{P}$ is nonempty, we say that $P$ is a solid cone.

Definition $2.3[3,5]$ : Let $(E, \tau)$ be a topological vector space and $P \subseteq E$ be a cone.

We define a partial ordering $\leq$ on $E$ with respect to $P$ by $x \leq y$ if and only if $y-x \in P$. We write $x<y$ if $x \leq y$ and $x \neq y$. Likewise, we write $x \ll \mathrm{y}$ if $\mathrm{y}-\mathrm{x} \in$ int $\mathrm{P}$, where int $\mathrm{P}$ denotes the interior of $\mathrm{P}$. If ambiguity is possible we can use the notations $\leq_{P},<_{P}$ and $\ll_{P}$. The pair $(E, P)$ consisting of a TVS $E$ and a solid cone $P$ of $E$ is called a partially ordered TVS.

In [2], it is proved that the existence of a solid cone in a TVS ensures that the vector topology is Hausdorff. In [1], it is mentioned that any TVS possessing a closed cone is Hausdorff. In view of [1] and from the assumption that $\mathrm{P}$ is both solid and closed, we observe that the proof in [2] is an extra effort.

Throughout this paper $(E, P)$ denotes a partially ordered real TVS E with a solid cone $P$.

Definition $2.4[3,5]$ Let $X$ be a nonempty set. A mapping $d: X \times X \rightarrow E$ satisfying

(d1) $d(x, y) \geq 0$;

(d2) $d(x, y)=0 \Leftrightarrow x=y$;

(d3) $d(x, y)=d(y, x)$ and

(d4) $d(x, y) \leq d(x, z)+d(z, y)$ for all $x, y, z \in X$,

is called a TVS-valued cone metric on $X$. The pair $(X, d)$ is called a TVS-valued cone metric space (written briefly as TVS-CMS).

Definition $2.5[3,5]$ Let $(X, d)$ be a TVS-cone metric space, $x \in X$ and $\left\{x_{n}\right\}, n=1,2, \ldots$ be a sequence in $X$. Then we say

i) $\left\{x_{n}\right\}$ TVS-converges to $\mathrm{x}$ if for every $\mathrm{c} \in$ int $\mathrm{P}$ there exists a natural number $\mathrm{N}$ such that $d\left(x_{n}, x\right) \ll c$ for all $n \geq N$.

ii) $\left\{x_{n}\right\}$ is a TVS-Cauchy sequence if for every $\mathrm{c} \in$ int $\mathrm{P}$ there exists a natural number $\mathrm{N}$ such that $\quad \mathrm{d}\left(\mathrm{x}_{\mathrm{n}}, \mathrm{x}\right.$ m) $\ll$ c for all $n, m \geq N$.

iii) $(X, d)$ is a TVS-complete cone metric space if every Cauchy sequence in $(X, d)$ is convergent in $(X, d)$.

Next we define the term algebra over a field and its concomitants.

Definition 2.6 [17]: An algebra A over a field $K$ is a vector space over $K$ such that for each ordered pair of elements $x, y \in A$ a unique product $x y \in A$ is defined with the properties

1) $(x y) z=x(y z)$;

2) $x(y+z)=x y+x z$ and $(x+y) z=x z+y z$ and

3) $\alpha(x y)=(\alpha x) y=x(\alpha y)$ for all $\alpha \in K$ and $x, y, z \in A$.

The algebra $A$ is called a real algebra if $\mathrm{K}=\mathbb{R}$ and a complex algebra if $\mathrm{K}=\mathbb{C}$.

Caution: In the literature, there are a few other variants of the definition of the term algebra over a field.

Definition 2.7 [17]: A topological algebra $A$ over a field $K$ is a topological vector space together with a continuous multiplication that makes $A$ an algebra over $K$.

We denote a topological algebra A with vector topology $\tau$ and multiplication “. " by $\left(\mathrm{A}, \tau,{ }^{\circ}\right)$. 
Two Fixed Point Theorems in Topological vector space Valued Cone Metric Spaces with Complete

Definition 2.8 [17]: A complete topological algebra A is a topological algebra which is complete, considered as a uniform space.

Definition 2.9 [5]: Let e $\in$ int $\mathrm{P}$. The formula $\xi_{e}(\mathrm{y}):=\inf \{\mathrm{t} \in \mathbb{R} \mid y \leq t e\}$, where $\mathrm{y} \in \mathrm{E}$, defines a function from $\mathrm{E}$ into $\mathrm{R}$, and is called the nonlinear scalarization function on $\mathrm{E}$ (w.r.t $\mathrm{P}$ and e).

The following lemma states properties of the nonlinear scalarization function $\xi_{e}$.

Lemma 2.10 [5, 2]: For any e $\in$ int $\mathrm{P}$, the function $\xi_{e}$ has the following properties:

1) $\xi_{e}(0)=0$

2) y $\in \mathrm{P}$ implies $\xi_{e}(\mathrm{y}) \geq 0$

3) If $\mathrm{y}_{2} \leq \mathrm{y}_{1}$, then $\xi_{e}\left(\mathrm{y}_{2}\right) \leq \xi_{e}\left(\mathrm{y}_{1}\right)$ for any $\mathrm{y}_{1}, \mathrm{y}_{2} \in \mathrm{E}$;

4) $\xi_{e}$ is subadditive on $\mathrm{E}$, i.e. $\xi_{\mathrm{e}}(\mathrm{x}+\mathrm{y}) \leq \xi_{\mathrm{e}}(\mathrm{x})+\xi_{\mathrm{e}}(\mathrm{y})$ for all $\mathrm{x}, \mathrm{y} \in \mathrm{E}$;

5) $\xi_{e}$ is positively homogeneous on $\mathrm{E}$, i.e., $\xi_{\mathrm{e}}(\beta \mathrm{x})=\beta \xi_{\mathrm{e}}(\mathrm{x})$ for every $\mathrm{x} \in \mathrm{E}$ and positive real number $\beta$, and

6) $\xi_{e}$ is continuous on $\mathrm{E}$.

Lemma 2.11[5, 2]: Let $(X, d)$ be a TVS-CMS over an ordered TVS $(E, P)$ and $e \in$ int $P$. Then

a) the function $d_{e}: E \times E \rightarrow[0, \infty)$ defined by $d_{e}=\xi_{e} o d$ is a metric.

b) $(X, d)$ is TVS-complete if and only if $\left(X, d_{e}\right)$ is complete.

\section{Main Results}

We start with introducing the notion of CTA cone.

Definition 3.1: Let $\left(\mathrm{E}, \tau,{ }^{-}\right)$be a real complete topological algebra with unity e. A subset $P$ of $E$ is called a complete topological algebra cone (briefly CTA cone) if $P$ is a cone of $E$ with the additional properties that:

(i) $e \in P$ and (ii) $x, y \in P$ implies $x y \in P$

Lemma 3.2: Let $(X, d)$ be a complete TVS-CMS with unity $e$ and $P \subseteq E$ be a CTA cone. Then the nonlinear scalarization function $\xi_{e}$ satisfies the following property:

$\xi_{e}(x y) \leq \xi_{e}(x) \xi_{e}(y)$ for all $x, y \in P$.

Proof: By definition, $\mathrm{x} \leq \xi_{e}(\mathrm{x})$ e. This implies $\xi_{e}(\mathrm{x}) \mathrm{e}-\mathrm{x} \in \mathrm{P}$. It follows from (3.1.1(ii)) and from the fact that $e$ is the unity of $\mathrm{E}$ that $\xi_{e}(\mathrm{x}) \mathrm{y}-\mathrm{xy} \in \mathrm{P}$. This in turn implies $\mathrm{xy} \leq \xi_{e}(x) \mathrm{y}$. On the other hand, $\xi_{e}(x) \mathrm{y} \leq \xi_{e}(x)$ $\xi_{e}$ (y) e. Using the transitivity property of the partial ordering $\leq$ we get $x y \leq \xi_{e}(x) \xi_{e}$ (y) e. Hence, the result follows.

The following theorem is an extension of the cone metric space version of Banach's Fixed point Theorem (i.e., Theorem 1 of [8] and Theorem 2.3 of [13]).

Theorem 3.3: Let $(X, d)$ be a complete TVS-CMS and $P \subseteq E$ be a complete topological algebra cone. Let $\mathrm{T}$ be a self mapping of $X$ satisfying

$d(T x, T y) \leq k d(x, y)$,

for some $\mathrm{k} \in \mathrm{P}$ with $0 \leq \mathrm{k} \ll \mathrm{e}$ and for all $\mathrm{x}, \mathrm{y} \in \mathrm{X}$. Then $\mathrm{T}$ has a unique fixed point.

Proof: From (3.2.1), Lemma 2.10(3) and Lemma 3.2 we get

$d(T x, T y) \leq k d(x, y) \Rightarrow \xi_{e}(d(T x, T y)) y \leq \xi_{e}(k d(x, y))$

$$
\Rightarrow d_{e}(T x, T y) \leq \xi_{e}(k) d_{e}(x, y)
$$

We show that $0 \leq \xi_{e}(k)<1$. From the assumption $0 \leq k \ll e$ and Lemma 2.10(3) it follows that $0 \leq \xi_{e}(k) \leq 1$. Suppose $\xi_{e}(k)=1$. Since $e-k \epsilon$ int $\mathrm{P}$, so there exists an open set $\mathrm{U}$ such that $e-k \epsilon U \subseteq$ int $P$. Since $k-e+U$ is a neighborhood of 0 and the sequence $\frac{-1}{n} e$ converges to 0 , so $-\beta e \in k-e+U$ for some $\beta>0$. Hence, $k \leq(1-\beta)$ $e$. This is a contradiction to the fact that $\xi_{e}(k)=1$. By Lemma 2.11(b) the metric space $\left(X, d_{e}\right)$ is complete and hence using (3.2.2) we can conclude that $\mathrm{X}$ has a unique fixed point.

Remark 3.4: If the cones used in [8], Theorem 1 and [13], Theorem 2.3 are CTA cones (so that $\mathrm{E}$ is a complete topological algebra), then these theorems are easy consequences of our Theorem 3.3 above. This can be justified as follows. Assume that the hypotheses of Theorem 2.3, [13] are true, i.e, let $(X, d)$ be a complete cone metric space with cone $P \subseteq E$. Let $\mathrm{T}$ be a self mapping of $\mathrm{X}$ satisfying

$d(T x, T y) \leq c d(x, y)$,

for some real number $\mathrm{c}$ such that $0 \leq \mathrm{c}<1$ and for all $x, y \in X$. Put $k:=c e$, where e is the unity of the T.V.S algebra E. Clearly $0 \leq k \ll e$ and $d(T x, T y) \leq k d(x, y)$. Thus the hypotheses of Theorem 2.3, [13] imply the hypotheses of Theorem 3.3 above. The rest is trivial.

Our next theorem is an extension of the cone metric space version of one of the Kannan type Fixed Point theorems [Theorem 2 [8] and Theorem 2.6 [13]].

Theorem 3.5 Let $(X, d)$ be a complete TVS-CMS and $P \subseteq E$ be a complete topological algebra cone. Let $\mathrm{T}$ be a self mapping of $X$ satisfying

$d(T x, T y) \leq k[d(T x, x)+d(T y, y)]$,

(3.5.1) 
Two Fixed Point Theorems in Topological vector space Valued Cone Metric Spaces with Complete for some $k \in P$; where $0 \leq \mathrm{k} \ll \frac{1}{2} e$ for all $\mathrm{x}, \mathrm{y} \in \mathrm{X}$. Then $T$ has a unique fixed point.

Proof: Using (3.5.1), Lemma 2.10 (3 and 4) and Lemma (3.2) we obtain

$d(T x, T y) \leq k[d(T x, x)+d(T y, y)] \Rightarrow \xi_{e}(d(T x, T y)) y \leq \xi_{e}(k[d(T x, x)+d(T y, y)])$

$$
\Rightarrow d_{e}(T x, T y) \leq \xi_{e}(k)\left[d_{e}(T x, x)+d_{e}(T y, y)\right]
$$

By assumption we have $0 \leq \mathrm{k} \ll \frac{1}{2} e$. This implies $0 \leq \xi_{e}(k) \leq \frac{1}{2}$. Suppose $\xi_{e}(k)=\frac{1}{2}$. Since $\frac{1}{2} e-k \in$ int $\mathrm{P}$, so there exists an open set $\mathrm{U}$ such that $\frac{1}{2} e-k \in \mathrm{U} \subseteq \mathrm{P}$. Since $\mathrm{k}-1 / 2 \mathrm{e}+\mathrm{U}$ is a neighbourhood of 0 and $\frac{-1}{n} e$ convergs to 0 , so there exists $\beta>0$ such that $-\beta \mathrm{e} \in \mathrm{W}$. This implies that $\left(\frac{1}{2}-\beta\right) e-k \in U \subseteq P$. Hence $\mathrm{k} \leq\left(\frac{1}{2}-\beta\right.$ le. This contradicts our supposition. Hence $0 \leq \xi_{e}(k)<\frac{1}{2}$. Since the TVS-CMS (X, d) is complete, so is the metric space $\left(X, d_{e}\right)$ (by Lemma 2.11(b)). The rest follows from Kannan's Fixed Point Theorem.

Remark 3.6 Theorem 3.5 is a generalization of the corresponding theorems in [8] and [13] provided that the cones used there in are CTA cones. The justification is analogous to that of Remark 3.4.

Remark 3.7: Theorems 3.3 and 3.5 give positive answers to one of the open problems raised in [15]. We insist that the conditions $\mathrm{k}<e$ and $k<\frac{1}{2} e$ cannot replace the corresponding conditions, $\|k\|<1$ and $\|k\|<\frac{1}{2}$, used in [15] in view of the following counter example.

Example 3.8: Let $X:=C[0,1]$ (the real vector space of all continuous real valued functions on $[0,1]$ equipped with the supremum norm). Then $\mathrm{X}$ is a real Banach space and $P=\{f \in X \mid 0 \leq f\}$ is a cone of X. See [14].

Moreover, int $P=\{f \in X \mid 0<f(x)$ for all $x \in[0,1]\}$ is nonempty. Thus $P$ is a solid cone.

Define $d: X \times X \rightarrow X$ by $d(f, g):=|f-g|$;i.e., $d(f, g):[0,1] \rightarrow \mathbb{R}$ given by $d(f, g)(x):=|f(x)-g(x)|, x \in X$. Note that $(X, d)$ is a complete CMS (hence a complete TVS-CMS) and the map Tf: $=k f+e$ (where $\mathrm{k}$ is the identity map on $[0,1]$ and $e x=1, x \in X)$ satisfies the conditions $f e=e f=e$ for all $\mathrm{f}$ in $\mathrm{X}, 0 \leq \mathrm{k}<\mathrm{e}$ and $d(T f, T g) \leq k d(f, g)$ for all $f$, $g$ in $X$ but $T$ has no fixed point. Hence the condition $k<e$ cannot replace the corresponding condition, $\|k\|<$ 1, used in Theorem 3.2[15]. By similar argument we can show that the condition $k<\frac{1}{2} e$ cannot replace the corresponding condition, $\|k\|<\frac{1}{2}$, used in Theorem 3.4[15].

Remark 3.9: In [2] the authors suggested that when contraction constants are taken from $P$, then fixed point statements in TVS-CMS may not be convertible to equivalent statements in usual metric spaces. But our results show that even when contraction constants are in $P$ the equivalences mentioned in [2] can be valid.

\section{Application}

Even though some fixed point statements stated in TVS-CMS can be converted to equivalent statements in usual metric spaces and vice versa, we suggest that it is still important to investigate fixed point theory of TVSCMS in greater depth. This is because unexpected applications can arise. For instance, it may happen that there can be cases where a fixed point problem posed in TVS-CMS is solvable with less effort than an equivalent problem posed in metric spaces or vice versa. We leave this as an open problem. If this happens to be true, then working in TVS-CMS can give us opportunity to solve more problems.

As an illustration we present an easy application to Theorem 3.3.

Example 4.1: Let $(X, d)$ be as described in Example 3.8. Define the operator $T$ by $(T f)(t):=\left(\frac{\sin t}{1+\sin t}\right) f(t), 0 \leq$ $t \leq 1$. Then $T$ is a self mapping of $X$. Moreover it is a contraction with respect to $d$ with "contraction constant" the function $k(t)=\sin t, 0 \leq t \leq 1$. Therefore $T$ has a unique fixed point.

\section{Conclusion}

In this paper and in [15] extensions of Banach's and Kannan's fixed point theorems to TVS-CMS (X, d) are discussed. In [15], $(X, d)$ is a cone metric space. The contraction constants $k$ used in both papers are no longer scalars but vectors. The results in these two papers can be summarized as follows. When $\|\mathrm{k}\|<1$ or $\mathrm{k} \ll \mathrm{e}$ ( for Banach's fixed point theorem) and $\|\mathrm{k}\|<1 / 2$ or $\mathrm{k} \ll 1 / 2 \mathrm{e}$ ( for Kannan's fixed point theorem), then the conclusions of the corresponding theorems are valid. But when $\|\mathrm{k}\|=1$ or $k<1$ (for Banach's fixed point theorem) and $\|\mathrm{k}\|=1 / 2$ or $\mathrm{k}<1 / 2 \mathrm{e}$ ( for Kannan's fixed point theorem), then the conclusions of the corresponding theorems are invalid.

\section{References}

[1] C.D. Aliprantis and R. Tourky, Cones and Duality (American Mathematical Society, 2007).

[2] I. D.Arandelovic and D. J. Keckic, TVS-Cone Metric Spaces as a Special case of Metric Spaces, arXiv: 1202.5930v1 [math.FA], 2012.

[3] I. Beg, A. Azam and M. Arshad, Common fixed points for maps on topological vector space valued cone metric spaces, Internat. J. Math. Math. Sciences, 2009 (2009).

[4] M. M. Deza and E. Deza, Encyclopedia of Distances (Springer-Verlag, 2009), i - x.

[5] W. S.Du, A note on cone metric fixed point theory and its equivalence, Nonlinear Analysis, 72 (5) (2010), $2259-2261$.

[6] M. Fréchet, Sur quelques points du calcul fonctionnel. Rendi. Circ. Mat. Palermo, 22(1906), 1- 74. 
[7] F. Hausdorff, Grundzüge der Mengenlehre, Verlag Von Veit \& Company, Leipzig (1914). Reprinted by Chelsea Publishing Company, New York (1949).

[8] L.G. Huang and X.Zhang, Cone Metric Spaces and Fixed Point Theorems of Contractive mappings, J. Math. Anal. Appl. , 332 (2007), $1467-1475$.

[9] M.C. Joshi and R.K. Bose, Some Topics in Nonlinear Functional Analysis (Wiley Eastern Ltd., New Delhi, 1985).

[10] D.R. Kurepa, Tableaux ramifies d'ensembles. Espaces pseudo-distancies, C. R. Acad. Sci. Paris, 198 (1934), 1563-1565.

[11] A.I. Perov, On Cauchy problem for a system of ordinary differential equations, Pviblizhen. Met. Reshen. Differ. Uvavn., 2(1964), $115-134$.

[12] P. D. Proinov, "A unified theory of cone metric spaces and its applications to the fixed point," In press, http://arxiv.org/abs/1111.4920.

[13] Sh. Rezapour, R.Hamlbarani, Some notes on the paper: Cone metric spaces and fixed point theorems of contractive mappings, J. Math. Anal. Appl. , 345 (2008), 719-724.

[14] W. Rudin, Functional Analysis (McGraw-Hill Science, 1991).

[15] K.P.R. Sastry, G.A. Naidu and Tadesse Bekeshie, Fixed Point Theorems in Cone Metric Spaces with Banach Algebra Cones, IJMSEA, (6)(V)(2012), $129-136$.

[16] J. S. Vandergraft, "Newton's method for convex operators in partially ordered spaces," SIAM Journal on Numerical Analysis, 4 (1967), 406-432.

[17] A. Wilansky, Modern Methods in Topological Vector Spaces (McGraw-Hill, 1978).

[18] P.P.Zabrejko, “K-metric and K-normed linear spaces: survey," Collectanea Mathematica, 48 (4-6) (1997), 825-859. 